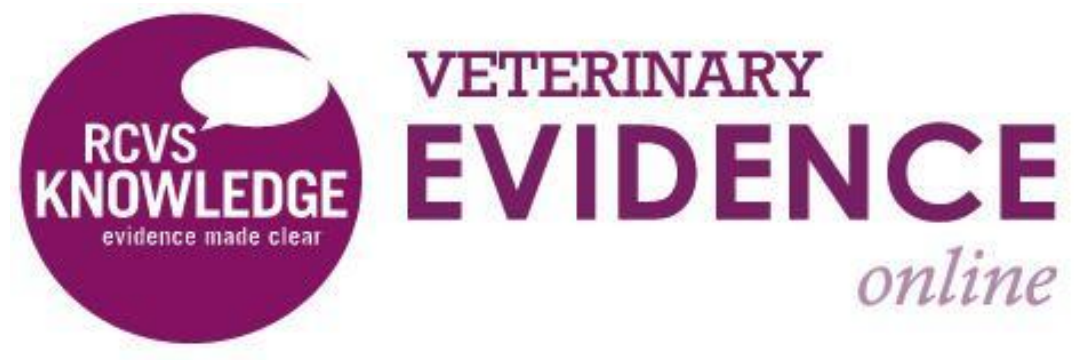

\title{
Use of a Reverse Thermodynamic Gel to Manage Chronic Shedding in Equine Strangles
}

Mark Bowen BVetMed MMedSci PhD CertVA CertEM(IntMed) DipACVIM-LAIM PFHEA FRCVS ${ }^{1 *}$

\footnotetext{
${ }^{1}$ Oakham Veterinary Hospital, Ashwell Road, Oakham LE15 7QH

Corresponding Author (Mark.Bowen@nottingham.ac.uk)
}

ISSN: 2396-9776

Published: 27 Jul 2017

in: Vol 2, Issue 3

DOI: http://dx.doi.org/10.18849/ve.v2i3.109

Reviewed by: Tim Mair (BVSc PhD DEIM DESTS DipECEIM AssocECVDI MRCVS) Kate McGovern (BVetMed CertEM(Int.Med) MS DACVIM DipECEIM MRCVS) 
Strangles is a common condition in equine practice with high morbidity. Management of outbreaks is complicated by the development of asymptomatic carriers with the causative organism (Streptococcus equi var equi) being retained within the guttural pouch ${ }^{1,2}$. Treatment of carriers relies upon removal of physical infection and locally administered antimicrobials. Combinations of penicillin with gelatin has been recommended ${ }^{3}$ to prolong antimicrobial concentrations within the guttural pouch.. This involves the melting of gelatin, to allow mixing and infusion of the warm liquid. The horses head is raised for 20 minutes while the solution sets as it cools, preventing loss of solution. These steps are often difficult to achieve outside of a hospital environment.

The availability of reverse thermodynamic agents, that are liquid when cool and solidify as they warm, have been used for local delivery of medicines when treating otitis externa in dogs. These create an opportunity to retain antibiotics in the guttural pouch without the complexity of melting gelatin in a field setting. Benzylpenicillin in poloxamer gel was specially formulated at a concentration of $120 \mathrm{mg} / \mathrm{ml}$ in a $25 \mathrm{ml}$ solution by a commercial specials manufacturer (BOVA UK). An asymptomatic strangles carrier was identified based on PCR detection of bacterial DNA from a guttural pouch lavage. After sedation, a stainless steel uterine catheter (Nielson catheter) was adapted by manually placing a 30 degree bend at the distal $3 \mathrm{~cm}$ tip of the catheter and approximately a 10 degree curve along the distal $15 \mathrm{cms}$ of the catheter (Figure 1 ). The catheter was placed along the ventral meatus with the tip angled ventrally. Once the catheter was in the pharynx, the tip was rotated ventrally and abaxially as the catheter was advanced into the guttural pouch; location was confirmed endoscopically (Figure 2). Cooled, pre-formulated poloxamer gel was infused through the urinary catheter and the catheter immediately removed. The head was maintained in a neutral position and a small amount of gel that exited the pouch as the catheter was removed, remained adherent to the pharyngeal wall demonstrating that it had already partially set.

Endoscopic assessment of the guttural pouch immediately after the infusion demonstrated that the poloxamer gel had partially set within the floor of the medial compartment of the treated pouch (observed 4 minutes 25 seconds after infusion: Figure 3). Treatment was repeated on three consecutive days and cure was determined by three guttural pouch lavages taken at weekly intervals, starting one week after the last treatment. This confirms the value of locally infused antibiotics in the management of strangles carriers. The reverse thermodynamic poloxamer gel provided a practical method for retaining antibiotics within the pouch with the need for complex mixing or prolonged raising of the horses head. This will facilitate management of this frustrating condition in the field. Administration through an endoscopic catheter is not recommended since the poloxamer gel partially solidifies within the biopsy channel due to the heat from the light source.

This case highlights the value of specially formulated medicines in the management of complex diseases by providing novel delivery vehicles.

\section{Figures}

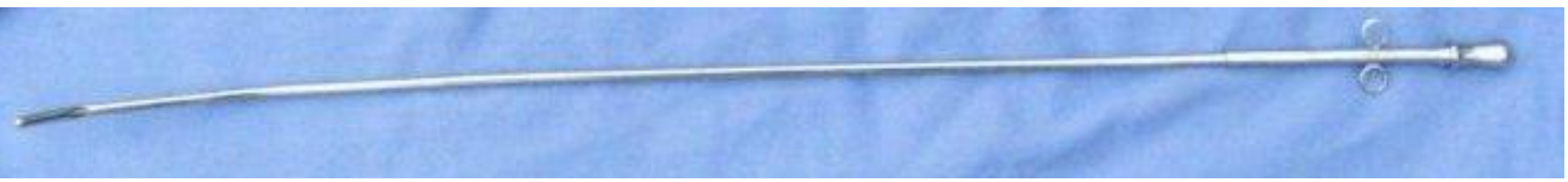

A 


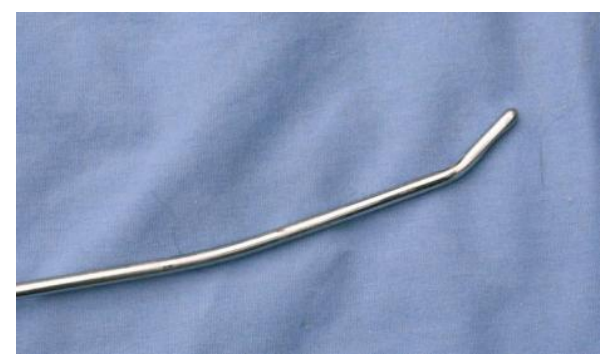

B

Figure 1: (A) The Nielson catheter that has been manually adapted to include a 30 degree distal bend at the tip and a 10 degree mid-shaft curve. (B) Shows a close up of the distal tip.

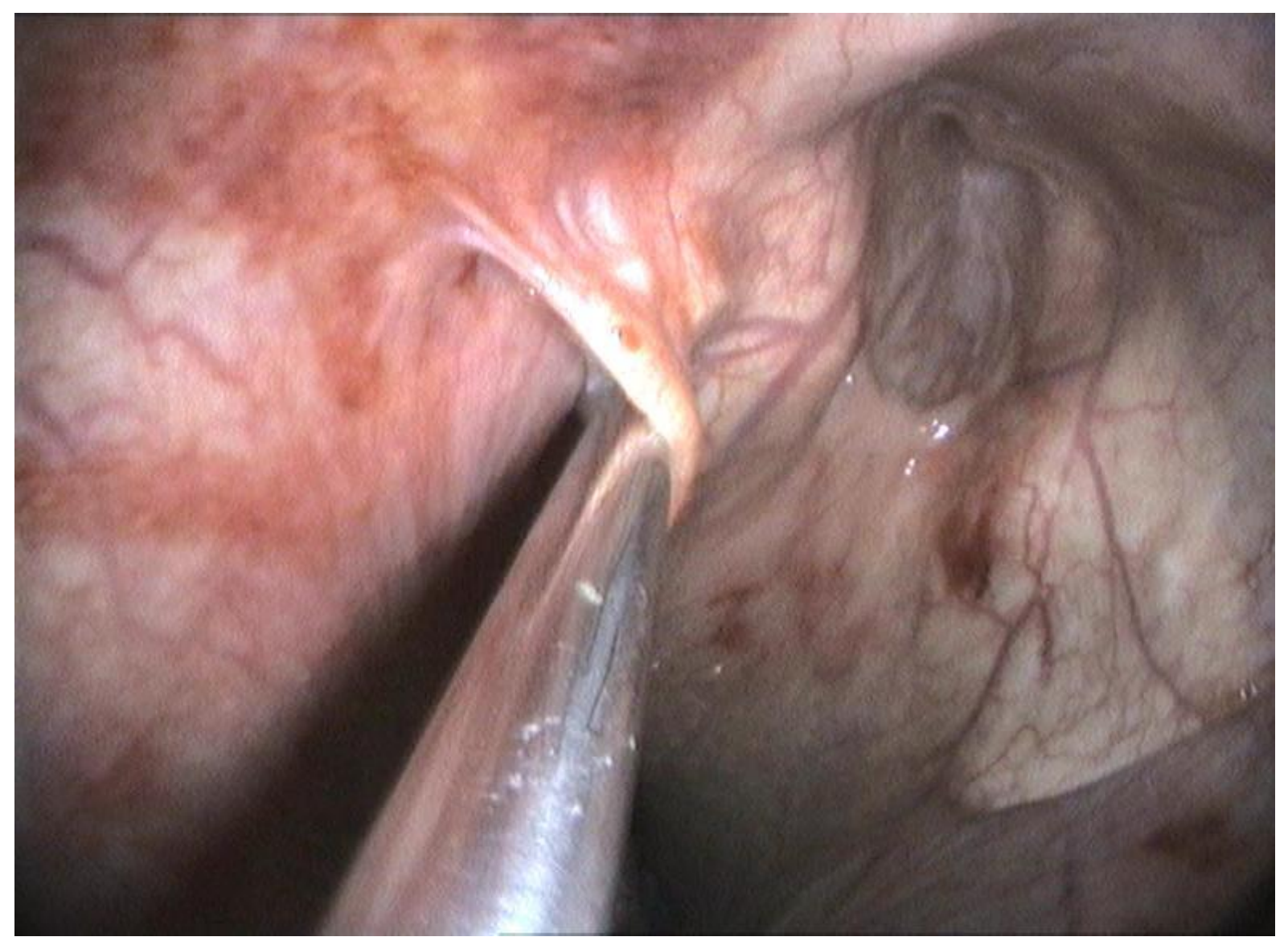

Figure 2: Endoscopic appearance of the pharynx showing the appearance of the Nielson catheter within the horse's right guttural pouch 


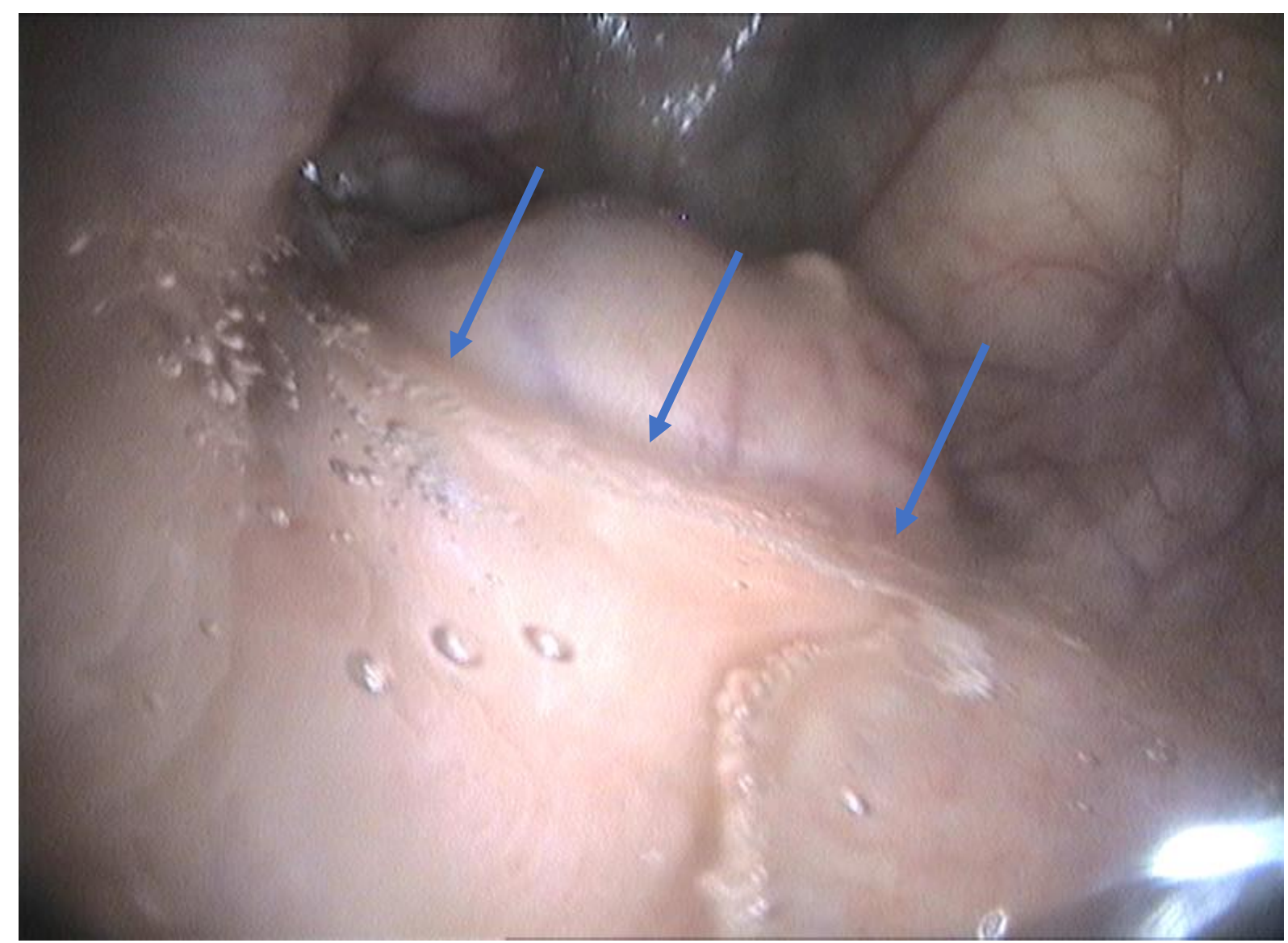

Figure 3: Endoscopic appearance of the partially solidified poloxamer gel 4 minutes 25 seconds after infusion into the guttural pouch of a strangles carrier. Lifiting of the head did not change the appearance of the meniscus within the pouch demonstrating it was already partially set. Arrows show meniscus of solidified gel against retropharyngeal mass (lymph node).

\section{Conflict of interest}

The author declares no conflict of interest.

\section{References}

1. George, J. L. et al. (1983) Identification of carriers of Streptococcus equi in a naturally infected herd. Journal of the American Veterinary Medical Association, 183 (1), pp. 80-84.

2. Newton, J. R., Verheyen, K., Talbot, N. C., Timoney, J. F., Wood, J. L. N., Lakhani, K. H. and Chanter, N. (2000), Control of strangles outbreaks by isolation of guttural pouch carriers identified using PCR and culture of Streptococcus equi. Equine Veterinary Journal, 32 (6), pp. 515526. http://dx.doi.org/10.2746/042516400777584721

3. Verheyen, K., Newton, J. R., Talbot, N. C., Brauwere, M. N. D. and Chanter, N. (2000), Elimination of guttural pouch infection and inflammation in asymptomatic carriers of Streptococcus equi. Equine Veterinary Journal, 32, pp. 527-532. doi:10.2746/042516400777584703 


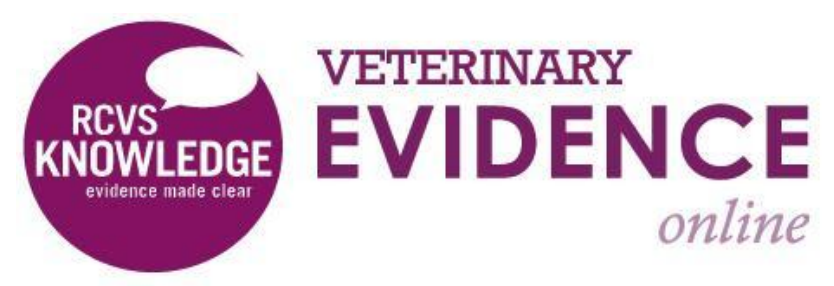

\begin{abstract}
Intellectual Property Rights
Authors of Articles submitted to RCVS Knowledge for publication will retain copyright in their work, but will be required to grant to RCVS Knowledge an exclusive license of the rights of copyright in the materials including but not limited to the right to publish, re-publish, transmit, sell, distribute and otherwise use the materials in all languages and all media throughout the world, and to license or permit others to do
\end{abstract} so.

Authors will be required to complete a license for publication form, and will in return retain certain rights as detailed on the form.

Veterinary Evidence and EBVM Network are RCVS Knowledge initiatives. For more information please contact us at editor@veterinaryevidence.org.

RCVS Knowledge is the independent charity associated with the Royal College of Veterinary Surgeons (RCVS). Our ambition is to become a global intermediary for evidence based veterinary knowledge by providing access to information that is of immediate value to practicing veterinary professionals and directly contributes to evidence based clinical decision-making.

www.veterinaryevidence.org

RCVS Knowledge is a registered Charity No. 230886. Registered as a Company limited by guarantee in England and Wales No. 598443.

Registered Office:

Belgravia House

62-64 Horseferry Road London SW1P 2AF

This work is licensed under a Creative Commons Attribution 4.0 International License. 